\title{
Two Pharmacological Phases in Antigen-Induced Immediate Airway Response in Rats
}

\author{
Naoki Miyagawa, ${ }^{*}$ Hidenori Iwasaki, Toshinobu Kato, Masaru Tanaka, Tsutomu Shibata, and \\ Korekiyo WAKITANI \\ Central Pharmaceutical Research Institute, Japan Tobacco, Inc.; 1-1 Murasaki-cho, Takatsuki, Osaka 569-1125, Japan. \\ Received August 24, 2008; accepted September 30, 2008; published online October 1, 2008
}

The pharmacological profiles of antigen-induced immediate airway response (IAR) in rats are not fully understood. In this study, we established an ovalbumin (OVA)-induced IAR model using noninvasive measurement in rats, and evaluated the effects of commonly used and effective antiasthmatic drugs, i.e. ketotifen (antihistamine), pranlukast (anti-leukotriene $C_{4} / D_{4} / E_{4}(L T)$ ), seratrodast (anti-thromboxane $A_{2}\left(T X A_{2}\right)$ ), salbutamol $(\beta 2$ agonist), and prednisolone (steroid). The rat IAR model exhibited an optimal rapid airway response, and salbutamol inhalation completely suppressed the IAR. Ketotifen inhibited only the quick phase (QP; the reaction from 3 to 6 min after challenge), while pranlukast and seratrodast suppressed only the early phase (EP; the reaction from 6 to $30 \mathrm{~min}$ after challenge). Prednisolone inhibited both QP and EP. Further, continuous administration of compound 48/80, which depletes connective tissue mast cells (CTMC), partially inhibited QP but not EP. In conclusion, these findings suggest that the pharmacological profiles of noninvasive rat IAR are similar to those of asthmatic patients, and that rat IAR exhibits additional, immunological diverse characteristics, i.e. QP caused by the exocytosis of mediators in CTMCs and EP mediated by LT and TXA, which are produced by mucosal mast cells (MMCs) and possibly by other types of cells. This is the first report about the comprehensive pharmacological profiles of rodent IAR model, and these analyses of rat IAR model may help expand our understanding of the diverse mechanisms underlying human asthmatic diseases.

Key words immediate airway response; ketotifen; pranlukast; salbutamol; prednisolone; compound 48/80

Allergic asthma is one of the most common respiratory diseases in the world, and asthmatic patients exhibit breathing difficulties classifiable into three types: specific antigeninduced immediate and late airway responses (IAR and LAR) and non-specific stimulus-induced airway hyperresponsiveness (AHR). ${ }^{1,2)}$ Of these three responses, IAR is sudden and strong and can be life-threatening in asthmatic patients.

The antigen-induced guinea pig IAR model has been reported to exhibit features similar to those of asthmatic patients, and has been widely used for pharmacological research. ${ }^{3,4)}$ On the other hand, since pharmaceutical targets for new asthmatic drugs have become more immunologically diverse and specific, detailed immunological analysis of mechanisms in animal models is required to determine with precision the efficacies of new drug candidates. However, the guinea pig airway model is not sufficient for this purpose, since immunological and genetic research on guinea pigs has not been performed in detail. Another alternative model such as a mouse or rat model is thus needed to determine the immunological effects of pharmaceuticals.

The involvement of mast cell mediators such as histamine, serotonin, and leukotriene $\mathrm{C}_{4} / \mathrm{D}_{4} / \mathrm{E}_{4}(\mathrm{LT})$ in rat IAR has been evaluated mainly in in vitro studies using isolated airway ${ }^{5}$ or invasive in vivo studies under anesthesia. ${ }^{6}$ In examining the pharmacological activities of new drug candidates, in vivo studies using noninvasive measurement are more useful and effective for prediction of effectiveness in humans. However, it has yet to be clearly determined whether clinical drugs exert effects on rat IAR using noninvasive measurement or how immunological mediators are involved in this rat IAR model.

In the present study, we established an ovalbumin (OVA)inhalation induced IAR model in rats using noninvasive measurement, and evaluated the effects of commonly used and effective antiasthmatic drugs to investigate the pharmacological characteristics of rat IAR.

\section{MATERIALS AND METHODS}

Animals Male Sprague-Dawley (SD) rats were purchased from Charles River Japan, Inc. (Yokohama, Japan) and individually housed in plastic cages maintained under specific pathogen-free conditions at a room temperature of $23.0 \pm 3.0^{\circ} \mathrm{C}$ and air humidity of $55 \pm 15 \%$ in a 12 -h light/ dark cycle environment. The rats were given a standard laboratory chow diet (CRF-1, Oriental Yeast, Tokyo, Japan) and water ad libitum. All experiments for animal use complied with the Guidelines of Animal Experimentation of our laboratories.

OVA-Induced Airway Responses SD rats were sensitized on day 0,1 and 2 by intraperitoneal (i.p.) injection of $10 \mu \mathrm{g}$ of OVA (Albumin from chicken egg white grade V, Sigma-Aldrich Co.) and $10 \mathrm{mg}$ of Alum (Imject Alum, PIERCE) in saline. On day 14, rats were challenged with exposure to aerosolized $0-5 \%(\mathrm{w} / \mathrm{v})$ OVA in saline for $5 \mathrm{~min}$. Aerosol challenge was performed with groups of up to 4 rats in a closed chamber attached to an ultrasonic nebulizer (NEU17; Omron Co., Tokyo, Japan). An indicator of airway response (Penh) was measured by barometric plethysmography using whole-body plethysmography (WBP; Buxco, Troy, NY, U.S.A.) under the pre-conditioned room temperature and humidity. ${ }^{7,8)}$ Penh values are a function of the sum of the airflows in the airways during a respiratory cycle. Penh has not yet been frequently measured in rats, ${ }^{9}{ }^{9}$ and potentially existing strain-related differences, such as described in mice. ${ }^{10,11)}$ However, Kirschvink et al. (2005) suggested by comparing Penh with $\mathrm{R}(\mathrm{L})$ that Penh can be used as an index of airflow 
limitation in spontaneously breathing under well-defined experimental condition in SD rats. ${ }^{12)}$ Rats were placed in the main chamber immediately after OVA-inhalation, and Penh values were continuously measured for $30 \mathrm{~min}$ and averaged for every $3 \mathrm{~min}$ period. Penh at $3 \mathrm{~min}$ indicated the average from 0 to $3 \mathrm{~min}$, and Penh $6 \mathrm{~min}$ indicated the average from 3 to $6 \mathrm{~min}$.

Compounds Ketotifen (Sigma-Aldrich Co.), pranlukast (Ono Pharmaceutical Co.), prednisolone (Sigma-Aldrich Co.), and seratrodast (Changzhou Huasheng Fine Chemical Co.) were prepared in $0.5 \%(\mathrm{w} / \mathrm{v})$ methylcellulose (MC) solution in distilled water, and administered $1 \mathrm{~h}$ before OVA challenge. A vehicle group was administered only $0.5 \% \mathrm{MC}$. The saline group consisted of sensitized rats administered $0.5 \% \mathrm{MC}$, which then inhaled aerosolized saline. Salbutamol (Wako, Osaka, Japan) was prepared $0.2 \%$ in saline, and inhaled via nebulizer by rats for $5 \mathrm{~min}$ before challenge. The vehicle group inhaled saline alone.

Pretreatment with Compound 48/80 To investigate the contribution of connective tissue mast cells (CTMC) to rat IAR, compound 48/80 (Sigma-Aldrich Co.) was preadministered to sensitized rats before challenge according to the method of Ikawati et al. ${ }^{5)}$ Briefly, sensitized rats were injected with compound 48/80 intraperitoneally from day 7 to day 13 at increasing doses ranging from an initial dose of $1 \mathrm{mg} / \mathrm{kg}$ to a final dose of $5 \mathrm{mg} / \mathrm{kg}$. Before each compound 48/80 injection, rats were also intraperitoneally administered mepyramine (Sigma-Aldrich Co.) at a dose of $10 \mathrm{mg} / \mathrm{kg}$ to prevent acute anaphylaxis.

Statistical Analysis All results are the means \pm standard error (S.E.). For the comparisons of two groups, the AspinWelch $t$-test (if data were unequal in variance) or Student $t$-test (if data were equal in variance) was used. Findings of $p<0.05$ were considered significant.

\section{RESULTS}

Dose-Response Relationship of Effects of OVA Inhalation on IAR Inhalational challenge of OVA $(0-5 \%)$ to sensitized rats increased Penh in dose-dependent fashion (Fig. 1). The airway response induced by each concentration of OVA peaked immediately after challenge. The 5\% OVA challenge strongly induced airway response and maintained it for $30 \mathrm{~min}$.

Effect of $\boldsymbol{\beta 2}$-Agonist on IAR Inhalational challenge of OVA $(5 \%)$ increased Penh in the vehicle group, exposed to aerosolized saline (Fig. 2). Exposure to salbutamol $(0.2 \%)$ before challenge completely eliminated antigen-induced airway response.

Effects of Anti-asthmatic Drugs on IAR Changes in Penh are shown in Figs. 3A and 4A. Ketotifen $(30 \mathrm{mg} / \mathrm{kg})$ decreased the airway response immediately after challenge (3-6 min) compared to vehicle group, while pranlukast $(30 \mathrm{mg} / \mathrm{kg})$ and seratrodast $(30 \mathrm{mg} / \mathrm{kg})$ did not. On the other hand, pranlukast and seratrodast decreased the airway response 6-30 min after challenge, while ketotifen did not. Prednisolone $(30 \mathrm{mg} / \mathrm{kg})$ decreased the both airway responses. To determine effects on total airway response, areas under the curve $(A U C)$ for 3-30 min were analyzed, as shown in Fig. 3B and Fig. 4B. Pranlukast, prednisolone, and seratrodast each significantly suppressed $A U C 3-30 \mathrm{~min}$,

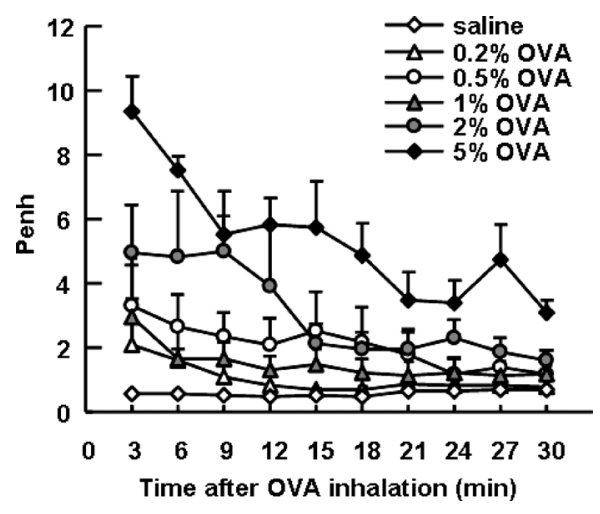

Fig. 1. Changes in IAR after Inhalation of OVA by Sensitized SD Rats

Actively sensitized rats inhaled several concentrations of OVA for $5 \mathrm{~min}$ using an ultrasonic nebulizer. After OVA inhalation, Penh was continuously measured by wholebody plethysmography for $30 \mathrm{~min}$ and averaged for 3-min periods. Values are the mean \pm S.E. $(n=4-8)$

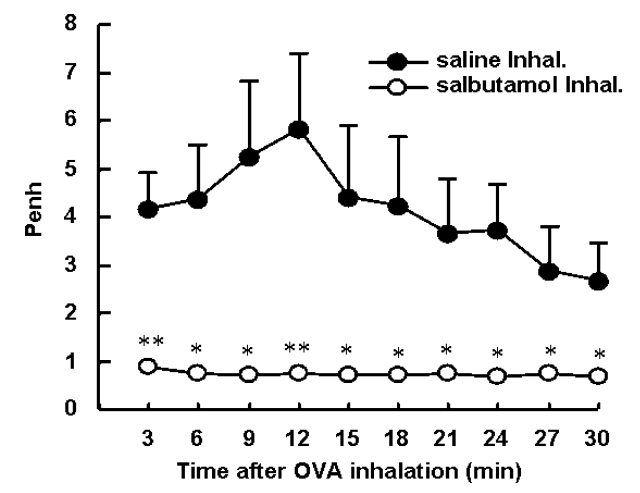

Fig. 2. Effects of Inhalation of $0.2 \% \mathrm{w} / \mathrm{v}$ Salbutamol Solution on Rat IAR

A salbutamol solution was inhaled for $10 \mathrm{~min}$ just before $5 \% \mathrm{w} / \mathrm{v}$ OVA inhalation. After OVA inhalation, Penh was continuously measured for $30 \mathrm{~min}$ and averaged over 3 -min periods. Values are the mean \pm S.E. $(n=6$ or 10$)$. $*, * * p<0.05,0.01$ (significantly different from saline inhalation group by Aspin-Welch $t$-test).

though ketotifen did not.

Based on the effects of these compounds, IAR was examined by separation into two phases, i.e. the quick phase (QP, $A U C$ 3-6 min) and early phase (EP, $A U C$ 6-30 min). $A U C$ analyses are shown in Figs. 3C, D and 4C, D. Ketotifen significantly suppressed QP but not EP, while pranlukast and seratrodast significantly suppressed EP but not QP. Only prednisolone suppressed both QP and EP.

Effect of Pretreatment with Compound 48/80 on IAR Changes in airway response after pretreatment with compound $48 / 80$ for $7 \mathrm{~d}$ were investigated to examine the contribution of CTMC to rat IAR. Pretreatment with compound 48/80 significantly decreased Penh only at 3 min after challenge (Fig. 5A). On $A U C$ analysis, pretreatment with compound 48/80 significantly decreased QP (AUC 3-6 min) but not EP ( $A U C 6-30 \mathrm{~min})$ (Figs. 5C, D).

\section{DISCUSSION}

In the present study, we examined an OVA-inhalation induced IAR model in rats using noninvasive measurement, and demonstrated that rat IAR has two unique time-dependent pharmacological phases, i.e. QP suppressed by ketotifen and compound 48/80 treatment, and EP suppressed by antileukotriene $\mathrm{C}_{4} / \mathrm{D}_{4} / \mathrm{E}_{4}(\mathrm{LT})$ and anti-thromboxane $\mathrm{A}_{2}\left(\mathrm{TXA}_{2}\right)$. 
A

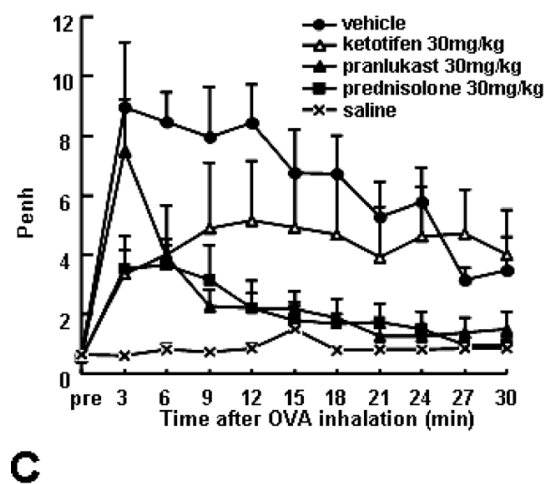

C

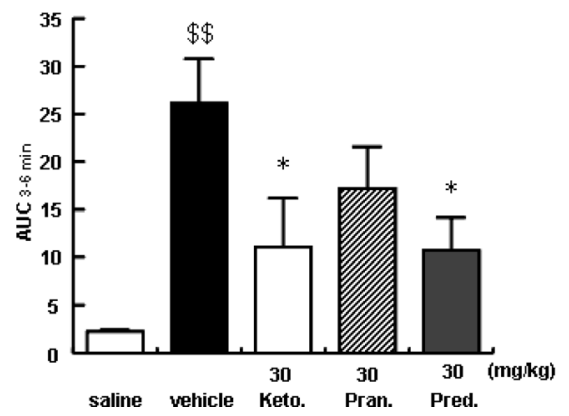

B

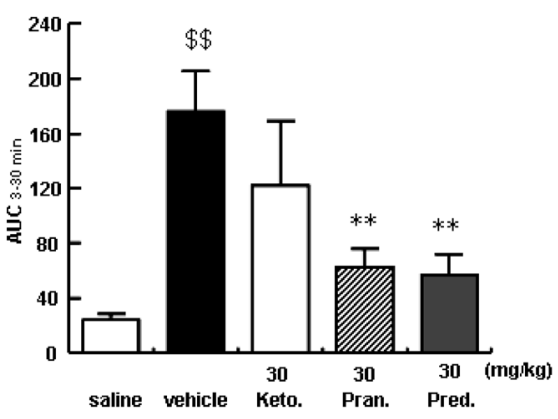

D

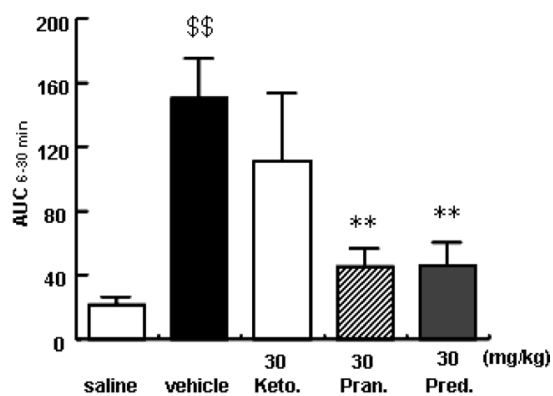

Fig. 3. The Effects of Ketotifen, Pranlukast, and Prednisolone on Rat IAR

One hour before $5 \%$ OVA inhalation, rats were orally administered ketotifen $30 \mathrm{mg} / \mathrm{kg}$, pranlukast $30 \mathrm{mg} / \mathrm{kg}$, or prednisolone $30 \mathrm{mg} / \mathrm{kg}$. Penh was continuously measured for 30 min and averaged over 3-min periods. (A) Time course changes after OVA inhalation. (B) $A U C$ from 3 to 30 min after OVA-inhalation. (C) $A U C$ from 3 to 6 min as an indicator of QP. (D) $A U C$ from 6 to 30 min as an indicator of EP. Values are the mean \pm S.E. ( $n=4$ or 13). Keto.: ketotifen, Pranl.: pranlukast, Pred.: prednisolone. $\$ \$ p<0.01$ (significantly different from saline group by Aspin-Welch $t$-test). $* * * p<0.05,0.01$ (significantly different from vehicle group by Student's $t$-test).

A

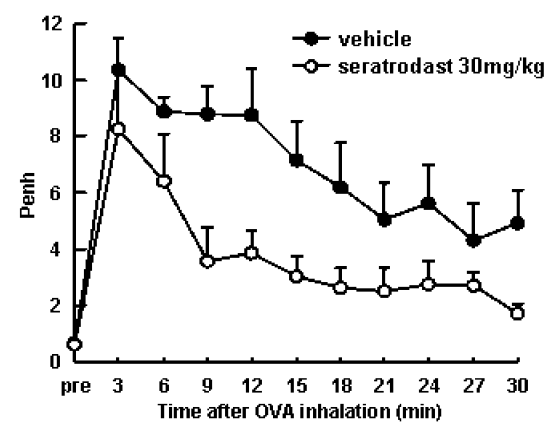

C

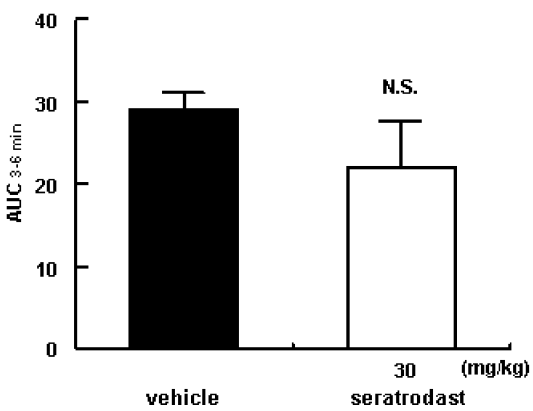

B

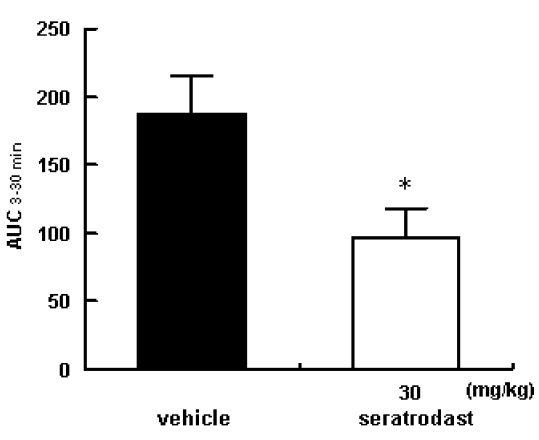

D

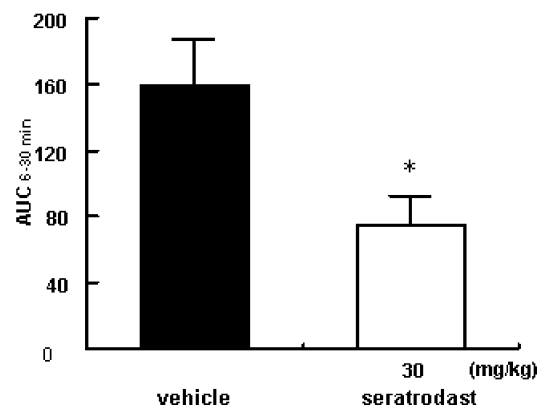

Fig. 4. The Effects of Seratrodast on Rat IAR

On day 14 , seratrodast $30 \mathrm{mg} / \mathrm{kg}$ was given orally $1 \mathrm{~h}$ before $5 \% \mathrm{w} / \mathrm{v}$ OVA inhalation. Penh was continuously measured for 30 min and averaged over 3 -min periods. (A) Time course changes after OVA inhalation. (B) $A U C$ from 3 to $30 \mathrm{~min}$ after OVA-inhalation. (C) $A U C$ from 3 to 6 min as an indicator of QP. (D) $A U C$ from 6 to 30 min as an indicator of EP. Values are the mean \pm S.E. $(n=4$ or 13$)$. $* p<0.05$ (significantly different from vehicle group by Student's $t$-test). N.S.: not significantly different. 
A

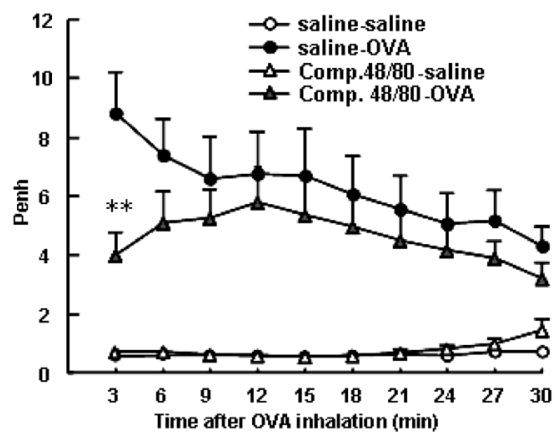

C

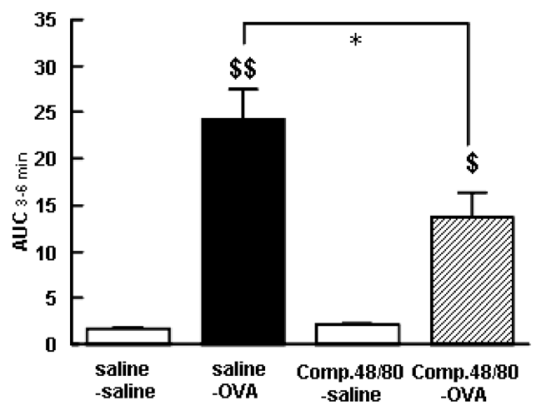

B

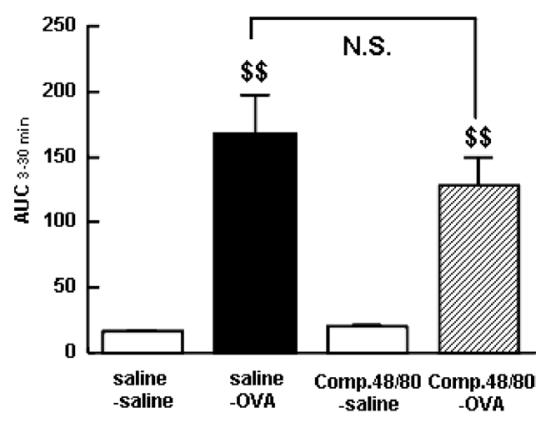

D

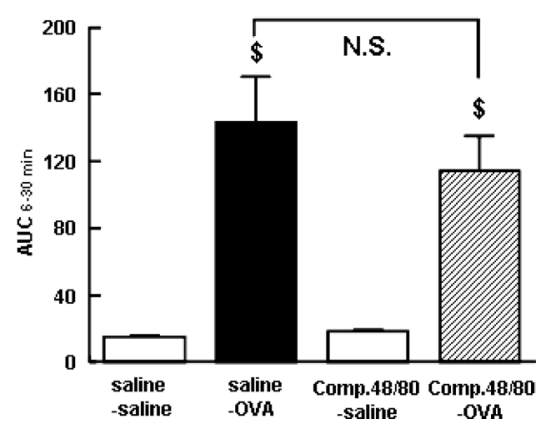

Fig. 5. The Effects of Pre-treatment of Compound 48/80 in Increasing Doses for $7 \mathrm{~d}$ before OVA Inhalation on Rat IAR

In the compound 48/80-pretreated group, increasing doses of compound 48/80 (1-5 mg/kg) were injected i.p. into sensitized rats from day 7 to 13 . In the saline-pretreated group, saline alone were injected. Thirty minutes before each saline or compound $48 / 80$ treatment, mepyramine $(10 \mathrm{mg} / \mathrm{kg})$ was administered i.p. to prevent lethal anaphylaxis. At day 14 , saline or $5 \% \mathrm{w} / \mathrm{v}$ OVA was inhaled and Penh was continuously measured for $30 \mathrm{~min}$ and averaged over 3 -min periods. (A) Time course changes after inhalation. (B) AUC from 3 to $30 \mathrm{~min}$ after inhalation. (C) $A U C$ from 3 to $6 \mathrm{~min}$ as an indicator of QP. (D) $A U C$ from 6 to $30 \mathrm{~min}$ as an indicator of EP. Values are the mean \pm S.E. ( $n=4$ or 13 ). Comp. 48/80: compound 48/80. $\$, \$ p<0.05,0.01$ (significantly different from each saline-inhalation group by Aspin-Welch $t$-test). $*, * * p<0.05,0.01$ (significantly different from respective saline-pretreated group by Student's $t$-test). N.S.: not significantly different.

Brown-Norway $(\mathrm{BN})$ rats have generally been used as models of asthma because their immunoglobulin E (IgE) production and eosinophil infiltrates are stronger than those of other rat strains. ${ }^{13,14)}$ Indeed, in our preliminary experiments, it was found that BN rats exhibited higher serum IgE production than SD rats after the same OVA treatment. As regards airway response, however, SD rats exhibited a stronger and quicker immediate response than $\mathrm{BN}$ rats. Because IAR is characterized by strong and quick airway responses in human asthma, the SD rat was chosen for use in this study.

Rat IAR has two different pharmacological phases: a QP, with a response 3 to $6 \mathrm{~min}$ after challenge, and an EP, with a response 6 to $30 \mathrm{~min}$ after challenge. QP was inhibited by ketotifen administration, suggesting that it is caused by the exocytosis of mediators (histamine, serotonin etc.) in mast cell since ketotifen has effects on histamine and mast cell degranulation. ${ }^{15)}$ On the other hand, EP was inhibited by pranlukast or seratrodast administration, suggesting that it is caused by LT and $\mathrm{TXA}_{2}$. Further, salbutamol almost completely suppressed both responses, suggesting that severe bronchoconstriction of the lower airway, but not the upper airway, is involved in QP and EP. Although the mechanism of inhibition by prednisolone of rat IAR is not fully understood, inhibition of smooth muscle contraction or LT/TXA 2 synthesis might affect airway responses. ${ }^{16,17)}$

In rodents, there are two types of mast cells, connective tissue type mast cells (CTMCs) and mucosal mast cells (MMCs). ${ }^{18)}$ These two types of cells differ not only in tissue distribution but also in pattern of mediator production. In rat airway, CTMCs and MMCs are observed in the submucosal region and epithelial layer, respectively. ${ }^{19,20)}$ Although both CTMCs and MMCs release histamine and serotonin, lipoxygenase products such as LT are predominantly generated by MMCs. ${ }^{21)}$ Ikawati et al. $(2000,2001)$ found in a histological study that when rats were administered compound 48/80 in increasing doses for $7 \mathrm{~d}$, tracheal CTMCs were almost completely eliminated, while the number of MMCs in trachea was unaffected. $^{5,20)}$ In our experiment, pretreatment with compound 48/80 diminished but did not eliminate QP, which was suppressed by ketotifen. On the other hand, compound 48/80 did not influence EP, which was suppressed by anti-LT and anti-TXA ${ }_{2}$. These findings are corresponded to the effects of compound 48/80 on $\mathrm{CTMC}^{5,20)}$ and with a report on the mediator profile of CTMC. ${ }^{21)}$ Summarizing findings, QP in rat IAR appears to be mediated by the exocytosis of mediators in CTMCs, while EP in rat IAR appears to be mediated by LT and TXA 2 , which are not generated by CTMCs.

Three patterns of antigen-induced responses have been found in asthmatics, i.e. isolated immediate asthmatic response (IAR), immediate and late response (Dual), and isolated late asthmatic response (LAR). ${ }^{22}$ The guinea pig asthmatic model exhibits two phases, IAR over a few minutes and LAR during a few hours after antigen exposure. ${ }^{23)} \mathrm{Re}-$ garding guinea pig IAR and the LAR model, it is known that antihistamine suppresses IAR but not LAR, ${ }^{24)}$ anti-TXA suppress IAR $^{25}$ ) and that anti-LT suppresses both IAR and LAR. $^{26)}$ In addition, $\beta 2$-agonists like salbutamol suppress only IAR but not LAR, indicating that IAR is mainly medi- 
ated by airway smooth muscle contraction. ${ }^{4)}$ The involvements of histamine, LT, TXA ${ }_{2}$, and smooth muscle contraction in rat IAR and guinea pig IAR are quite similar.

In conclusion, we have shown that rat IAR has immunologically diverse characteristics, i.e. two unique pharmacological profiles. QP might be caused by the exocytosis of mediators (histamine, serotonin etc.) in CTMCs and EP by LT and $\mathrm{TXA}_{2}$, which are produced by MMCs and possibly by other types of cells. This is the first report about the comprehensive pharmacological profiles of rodent IAR model, and the further analysis of rat IAR model may help expand our understanding of the diverse mechanisms underlying human asthmatic diseases.

Acknowledgement We thank Hiroyuki Tsuji for excellent advice.

\section{REFERENCES}

1) Cockcroft D. W., Can. Respir J., 5 (Suppl. A), 14A—17A (1998).

2) Larsen G. L., Clin. Immunol. Immunopathol., 53, S107-S118 (1989).

3) Evans T. W., Rogers D. F., Aursudkij B., Chung K. F., Barnes P. J., Am. Rev. Respir. Dis., 138, 395-399 (1988).

4) Matsumoto T., Ashida Y., Tsukuda R., J. Pharmacol. Exp. Ther, 269, 1236-1244 (1994).

5) Ikawati Z., Hayashi M., Nose M., Maeyama K., Eur. J. Pharmacol., 402, 297-306 (2000).

6) Hele D. J., Birrell M. A., Webber S. E., Foster M. L., Belvisi M. G., Br. J. Pharmacol., 132, 481-488 (2001).

7) Hamelmann E., Schwarze J., Takeda K., Oshiba A., Larsen G. L., Irvin C. G., Gelfand E. W., Am. J. Respir. Crit. Care Med., 156, 766-775 (1997).

8) Elekes K., Helyes Z., Kereskai L., Sándor K., Pintér E., Pozsgai G., Tékus V., Bánvölgyi A., Németh J., Szuts T., Kéri G., Szolcsányi J., Eur. J. Pharmacol., 578, 313-322 (2008).
9) Zhang X. D., Andrew M. E., Hubbs A. F., Siegel P. D., Toxicol. Sci., 94, 322-329 (2006)

10) Adler A., Cieslewicz G., Irvin C. G., J. Appl. Physiol., 97, 286-292 (2004).

11) Duguet A., Biyah K., Minshall E., Gomes R., Wang C. G., TaoudiBenchekroun M., Bates J. H., Eidelman D. H., Am. J. Respir. Crit. Care Med., 161, 839-848 (2000).

12) Kirschvink N., Vincke G., Onclinx C., Peck M. J., Gustin P., J. Pharmacol. Toxicol. Methods, 51, 123-128 (2005).

13) Singh P., Daniels M., Winsett D. W., Richards J., Doerfler D., Hatch G., Adler K. B., Gilmour M. I., Am. J. Physiol. Lung Cell. Mol. Physiol., 284, L588-L598 (2003).

14) Hylkema M. N., Hoekstra M. O., Luinge M., Timens W., Clin. Exp. Immunol., 129, 390-396 (2002).

15) Martin U., Römer D., Arzneimittelforschung, 28, 770-782 (1978).

16) Emala C. W., Clancy J., Hirshman C. A., Am. J. Physiol., 272, L745L751 (1997).

17) Peters-Golden M., Thebert P., Am. Rev. Respir. Dis., 135, 1020-1026 (1987).

18) Aldenborg F., Enerbäck L., Histochem. J., 20, 19-28 (1988).

19) Tam E. K., Calonico L. D., Nadel J. A., McDonald D. M., Anat. Embryol., 178, 107-118 (1998).

20) Ikawati Z., Nose M., Maeyama K., Jpn. J. Pharmacol., 86, 38-46 (2001).

21) Heavey D. J., Ernst P. B., Stevens R. L., Befus A. D., Bienenstock J., Austen K. F., J. Immunol., 15, 1953-1957 (1988)

22) Satoh A., Munakata M., Amishima M., Nasuhara Y., Honmna Y., Kawakami Y., Nihon Kyobu Shikkan Gakkai Zasshi, 33, 612-617 (1995).

23) Nagao K., Akabane H., Masuda T., Komai M., Tanaka H., Nagai H., J. Pharm. Pharmacol., 56, 187-196 (2004).

24) Justesen D. R., Braun E. W., Garrison R. G., Pendleton R. B., Science, 170, 864-866 (1970).

25) Saito M., Fujimura M., Ogawa H., Matsuda T., J. Lipid Mediat. Cell Signal., 11, 1-12 (1995).

26) Nakagawa N., Obata T., Kobayashi T., Okada Y., Nambu F., Terawaki T., Furuya T., Muryobayashi K., Sawada M., Aishita H., Eur. J. Pharmacol., 235, 211-219 (1993). 